\title{
Indicação inicial de tratamento em 60 pacientes com distúrbios ventilatórios obstrutivos do sono
}

\author{
Paulo de Tarso Moura Borges ${ }^{1}$ \\ Jorge Rizzato Paschoal
}

\section{Initial indication of treatment in 60 patients with sleep obstructive ventilatory disturbance}
Palavras-chave: apnéia, síndromes da apnéia do sono, apnéias do sono tipo obstrutivas.
Key words: apnea, sleep apnea syndromes, sleep apnea, obstructive.

Resumo / Summary

$\mathbf{O}$ bjetivo: Os autores apresentam um estudo descritivo retrospectivo de 60 pacientes portadores de distúrbios ventilatórios obstrutivos do sono (DVOS), atendidos no Centro Campinas de Otorrinolaringologia e Cirurgia de Cabeça e Pescoço num período de três anos. Todos os pacientes foram examinados segundo protocolo padronizado e as decisões quanto à primeira conduta terapêutica resultaram de discussão conjunta multidisciplinar sistemática. Forma de estudo: clínico retrospectivo. Material e método: Os pacientes foram distribuídos em dois grupos segundo a proposta de tratamento não-cirúrgico e cirúrgico. Em seguida, foram estudados quanto à modalidade inicial de tratamentos propostos e os principais achados propedêuticos: índice de distúrbio respiratório (IDR), índice de massa corpórea (IMC), análise cefalométrica e manobra de Müller. Os principais achados propedêuticos foram comparados, isoladamente ou em associações com a modalidade de tratamento proposto. Conclusão: As principais conclusões mostram que nas roncopatias, a indicação de tratamento não-cirúrgico e cirúrgico se fez na mesma proporção; a indicação de tratamento cirúrgico prevaleceu na Síndrome da Apnéia-Hipopnéia Obstrutiva do Sono (SAHOS), independente de sua modalidade; o IDR, o IMC e a manobra de Müller não tiveram influência na indicação de qualquer modalidade terapêutica; a decisão terapêutica decorreu de estudo propedêutico sistematizado e da atuação multidisciplinar, havendo cada caso sido discutido individualmente.

\begin{abstract}
A
im: The author present a retrospective descriptive study of 60 patients with sleep obstructive ventilatory disturbance who have taken medical advice at the Centro Campinas de Otorrinolaringologia e Cirurgia de Cabeça e Pescoço during a period of three years. All the patients have been examined after standardized protocol and decisions related to the treatment have been taken after systematic multidisciplinary discussion. Study design: clinical retrospective. Material and method: The patients were distributed into two groups according to the proposal of surgical and non-surgical treatment. After so, they were studied according to the model of treatment proposed and the main propaedeutic findings: respiratory disturbance index (RDI), body mass index (BMI), cephalometric analysis and Müller maneuver. The main features were compared - isolated or in association - with the model of treatment proposed. Conclusion: Amongst several conclusions obtained, the most important were: surgical and non-surgical treatment were indicated almost in the same proportion for of snoring; surgical treatments were most indicated for snoring and Apnoea-Hipopnoea Syndrome, despite of its modality; RDI, BMI and cephalometric analysis and Müller maneuver had no influence at any therapeutic modality; the therapeutic decision was taken after standardized protocol and systematic multidisciplinary discussion, where each case was discussed individually.
\end{abstract}

\footnotetext{
${ }^{1}$ Mestrado em Medicina pela UNICAMP, Professor Assistente da Disciplina de Otorrinolaringologia da Universidade Federal do Piauí. ${ }^{2}$ Doutor em Otorrinolaringologia pela UNICAMP, Professor Assistente da Disciplina de Otorrinolaringologia da UNICAMP. Faculdade de Ciências Médicas da Universidade Estadual de Campinas (UNICAMP).

Endereço para correspondência: Paulo de Tarso Moura Borges - Av. Elias João Tajra 1260 apto. 300 Jóquei Clube Teresina Piauí 64.049-300. Este artigo foi submetido no SGP (Sistema de Gestão de Publicações) da RBORL em 5/6/2005 e foi aprovado em 6/9/2005. Artigo recebido em 05 de junho de 2005. Artigo aceito em 6 de setembro de 2005 .
} 


\section{INTRODUÇÃO}

O diagnóstico e a conduta terapêutica nos distúrbios ventilatórios obstrutivos do sono (DVOS) têm sido apresentados na literatura como um desafio conseqüente à fisiopatologia multifatorial ${ }^{1}$. O espectro da Síndrome da Apnéia-Hipopnéia Obstrutiva do Sono (SAHOS) pode variar desde ronco, com interferência apenas social, até situações graves de apnéia com êxito letal ${ }^{2}$. A SAHOS acomete $4-7 \%$ da população geral adulta ${ }^{3}$. Pela sua prevalência, hoje é considerada uma preocupação pública maior, com graves conseqüências físicas e sociais se não tratada adequadamen$\mathrm{te}^{3,4}$.

Esses distúrbios afetam principalmente pacientes de meia idade, profissionalmente ativos, acarretando altos custos e perdas de dias de trabalho5. Os custos médicos dos DVOS podem ser reduzidos significativamente quando o diagnóstico e o tratamento efetivos são realizados precocemente ${ }^{6}$.

As diversas modalidades de tratamento propostas para o ronco e a SAHOS podem envolver a participação de profissionais de várias áreas ${ }^{7}$ e devem ser adaptadas às características individuais do paciente e à natureza da obstrução. A opção pelos diversos tipos de tratamento depende da história do paciente, da morbidade do processo e dos respectivos efeitos colaterais e benefícios destes procedimentos terapêuticos ${ }^{8}$.

Além da origem multifatorial dos distúrbios ventilatórios obstrutivos do sono, que muitas vezes dificulta o diagnóstico preciso dos fatores relacionados à obstrução e a sua correção, a maioria dos pacientes tem dificuldade na adesão e no seguimento do tratamento adequado. Como o grande número destes pacientes é obeso e muitos cultivam o hábito de ingerir bebida alcoólica, de alimentarem-se antes de deitar ou só conseguirem dormir em decúbito dorsal, a maior dificuldade reside na mudança do estilo de vida, evitando-se todos esses fatores agravantes. Muitos iniciam o tratamento, desistindo em seguida.

Muitos pacientes não aceitam tratamento cirúrgico, principalmente os procedimentos mais complexos. Mesmo esses tratamentos só são efetivos quando associados ao tratamento comportamental.

O objetivo do presente trabalho é identificar a influência da polissonografia, do índice de massa corpórea, da cefalometria e da manobra de Müller na indicação inicial de tratamento de um grupo de pacientes portadores de distúrbios ventilatórios obstrutivos do sono e rever os procedimentos mais indicados.

\section{MATERIAL E MÉTODO}

Foram estudados, retrospectivamente, os dados obtidos de prontuários de 60 pacientes de ambos os sexos, na faixa etária entre 19 a 70 anos. Os pacientes advinham de amostragem seqüencial e não-selecionada que procuraram atendimento otorrinolaringológico no Centro Campinas de Otorrinolaringologia e Cirurgia de Cabeça e Pescoço, com queixas de ronco, sonolência diurna excessiva ou sono nãoreparador, no período compreendido entre janeiro de 1998 a dezembro de 2001.

Os prontuários de pacientes que apresentavam essas queixas, mas que manifestavam deformidades craniofaciais ou eram portadores de doenças rinossinusais e/ou de faringe, tais como tumores, pólipos e cistos, foram excluídos do estudo.

O estudo foi realizado independentemente de sexo e de faixa etária.

Todos os pacientes haviam sido submetidos à avaliação clínico-otorrinolaringológica segundo protocolo padronizado, contendo dados de anamnese e exame físico.

O índice de massa corpórea (IMC) havia sido calculado em todos os pacientes. IMC é a relação entre o peso do indivíduo em quilogramas e a altura em metros quadrados. Considerou-se obeso o paciente com IMC $>27,3$.

Semiologia complementar empregada:

1. Vídeo-fibro-naso-faringo-laringoscopia, com avaliação estática e dinâmica das vias aéreas superiores, da cavidade nasal à laringe, com pesquisa da manobra de Müller. Esta manobra foi realizada na rinofaringe e na orofaringe/ hipofaringe, para observar a ocorrência de estreitamento nestas regiões. É considerada positiva quando ocorre estreitamento e negativa quando não ocorre.

2. Polissonografia de noite inteira, segundo padronização;

3. Telerradiografia em perfil, com análise cefalométrica, considerando-se as seguintes medidas lineares na avaliação das vias aéreas superiores: espaço aéreo posterior (PAS), definido como o espaço atrás da língua e limitado por tecidos moles; distância da espinha nasal posterior até a borda posterior do palato mole (PNS-P), que determina o comprimento do palato mole; distância do plano mandibular ao osso hióide (MP-H) ${ }^{9,10}$. Utilizamos como parâmetros comparativos os valores considerados normais por Riley et al. ${ }^{9}:$ PAS $\geq 11 \pm 1 \mathrm{~mm}, \mathrm{Mp}-\mathrm{H} \leq 15,4 \pm 3 \mathrm{~mm}$ e PNS-P $\leq 37 \pm$ $3 \mathrm{~mm}$, embora sujeitos às críticas decorrentes de inexistência de um padrão cefalométrico brasileiro, tendo em vista a complexidade de nossa composição racial.

Os prontuários foram divididos em quatro grupos, segundo classificação em roncopatia, SAHOS leve, SAHOS moderado e SAHOS grave ${ }^{11}$ :

Grupo 1: de pacientes com roncopatia;

Grupo 2: de pacientes com SAHOS leve;

Grupo 3: de pacientes com SAHOS moderado;

Grupo 4: de pacientes com SAHOS grave.

A distinção entre pacientes com roncopatia ou com SAHOS se fez por meio de avaliação individual da 
polissonografia, fundamentada no índice de apnéia e hipopnéia (IAH), que soma o número de eventos respiratórios por hora de sono.

Pacientes com IAH $<5$ eventos por hora de sono foram considerados como portadores de roncopatia; pacientes com IAH entre 5 e 15 eventos por hora de sono, portadores de SAHOS leve; pacientes com IAH acima de 15 e abaixo de 30 eventos por hora de sono, portadores de SAHOS moderado; pacientes com IAH acima de 30 eventos por hora de sono, portadores de SAHOS grave ${ }^{10}$.

Os pacientes foram divididos em 2 subgrupos, quanto à indicação de tratamento: tratamento não-cirúrgico e tratamento cirúrgico. O tratamento não-cirúrgico incluiu: mudanças comportamentais, Aparelhos Intra-Orais (AIO), tratamento medicamentoso e CPAP. Mudanças comportamentais em nosso trabalho incluem perda de peso, abstenção de ingestão de álcool e mudança de decúbito. Os tratamentos medicamentosos utilizados foram o antidepressivo tricíclico e a aminofilina.

Como tratamento cirúrgico foram indicados: uvulopalatofaringoplastia (UPFP), UPFP associado à AIO, laserassisted uvulopatoplasty (LAUP), LAUP associado à AIO, LAUP associado à septoplastia, LAUP associado à septoplastia e turbinectomia, avançamento mandibular associado à UPFP, septoplastia associado à uvulectomia, traqueostomia, uvulectomia, levantamento do hióide e amigdalectomia.

Estudou-se a modalidade de tratamento inicial indicada em cada subgrupo, em relação ao tipo de distúrbio respiratório (ronco, SAHOS leve, moderada e grave), ao IMC (< 27,3 e $\geq 27,3$ ), às medidas cefalométricas (PAS, PNS-P e $\mathrm{Mp}-\mathrm{H})$ e à manobra de Müller positiva na rinofaringe e/ou orofaringe/hipofaringe. Só foram considerados os prontuários dos pacientes que haviam sido submetidos a todos estes exames no período citado.

O estudo é de caráter descritivo.

O projeto foi aprovado previamente pelo Comitê de Ética em Pesquisa da Faculdade de Ciências Médicas da UNICAMP.

\section{RESULTADOS}

O grupo com indicação de tratamento não-cirúrgico compreendeu prontuários de 19 pacientes distribuídos segundo o distúrbio ventilatório em: 5 pacientes com roncopatia, 7 com SAHOS leve, 2 com SAHOS moderada e 5 com SAHOS grave.

O tratamento não-cirúrgico mais indicado nos casos de roncopatia foi orientação de mudanças comportamentais, com 3 (60\%) indicações. Para os pacientes com SAHOS leve, indicamos AIO em $4(57,14 \%)$. Naqueles com SAHOS moderada, AIO e tratamento medicamentoso foram os procedimentos mais indicados, com indicação igual em ambos os grupos. Nos casos de SAHOS grave, o mais indicado foi CPAP, com 2 indicações (40\%).
O grupo com indicação de tratamento cirúrgico constou de 41 pacientes, distribuídos segundo o distúrbio ventilatório em 6 pacientes com roncopatia, 13 com SAHOS leve, 6 com SAHOS moderada e 16 com SAHOS grave.

Uvulectomia foi a cirurgia mais indicada nos pacientes com roncopatia, com 3 indicações (50\%). Nos grupos com SAHOS leve, moderada e grave, UPFP foi o procedimento mais indicado, com 9 (69,23\%), 3 (50\%) e 11 (68,75\%) indicações, respectivamente.

Com relação ao tratamento mais indicado, em relação ao IMC, AIO foi o procedimento não-cirúrgico predominantemente recomendado a pacientes com valores inferiores a 27,3 (5 pacientes $=$ com 55,55\%). Mudança comportamental foi o tratamento mais indicado em pacientes com valores iguais ou superiores a 27,3 $(4$ pacientes $=$ $40 \%)$.

UPFP foi o procedimento cirúrgico mais indicado tanto em pacientes com IMC $<27,3(14$ pacientes $=56 \%)$ como em pacientes com IMC $\geq \mathrm{a} 27,3$ (11 pacientes $=44 \%)$. Em relação às medidas cefalométricas, mudanças comportamentais foram o procedimento não-cirúrgico mais indicado em pacientes com PAS $<11$ ( 7 pacientes $=43,74 \%)$. Os pacientes se distribuíram, conforme o distúrbio ventilatório, em 3 com roncopatia, 3 com SAHOS leve e 1 com SAHOS grave. Três apresentaram IMC $<27,3$, sendo 2 com roncopatia e 1 com SAHOS leve. Os demais, obesos, distribuíram-se em um com roncopatia, 2 com SAHOS leve e 1 com SAHOS grave. AIO foi o tratamento mais indicado em pacientes com PAS $\geq 11$ (dois pacientes $=66,67 \%$ ). Um desses pacientes, com SAHOS leve, era obeso e o outro, com roncopatia, apresentava IMC $=24$. Todos os pacientes apresentaram PNS-P $\geq 37$ e somente 2 apresentaram Mp-H $<15,4$, ou seja, praticamente todos com esses valores cefalométricos acima dos padrões normais na literatura. Portanto, essas medidas não foram consideradas em nosso estudo.

UPFP foi a cirurgia mais indicada quando se considerou o PAS, independentemente da sua variação: 13 pacientes com PAS $<11(31,71 \%)$ e 12 pacientes com PAS $\geq 11$ $(29,27 \%)$.

Os 12 pacientes com PAS $\geq 11$ distribuíram-se em: 2 com roncopatia, sendo 1 obeso; 5 com SAHOS leve, sendo 1 obeso; 5 com SAHOS grave, sendo 3 obesos.

\section{DISCUSSÃO}

A fisiopatologia multifatorial nos distúrbios ventilatórios obstrutivos do sono caracteriza um desafio para o seu tratamento $^{1}$. Estes distúrbios compreendem o ronco e a SAHOS, com suas diferentes modalidades. O tratamento baseia-se nas alterações anatômicas e clínicas, no grau de severidade da doença e nos fatores de risco ${ }^{7,12,13}$. A experiência do especialista em distúrbios do sono é fundamental para o sucesso na resolução desses problemas, sem dúvida. Todas as 
opções terapêuticas devem ser discutidas com o paciente, focalizando-se benefícios e insucessos possíveis ${ }^{14}$. A preferência do paciente pelas opções terapêuticas tem papel relevante no processo. O tratamento deve sempre envolver a participação de profissionais de várias áreas? .

Como o exame físico não é sensível nem específi$\mathrm{CO}^{15}$, em nosso serviço seguimos sempre uma rotina constituída de anamnese dirigida, vídeo-fibro-naso-faringolaringoscopia, cefalometria e polissonografia. Todos os exames são estudados por uma equipe multidisciplinar composta de otorrinolaringologista, neurologista, cirurgiã bucomaxilo-facial e fonoaudióloga, e a decisão terapêutica é tomada em conjunto.

A manobra de Müller é realizada com o paciente acordado. Embora se tente simular o que ocorre durante o sono, pode-se incorrer em interpretações errôneas, uma vez que o paciente acordado não apresenta o relaxamento do Comando central próprio dele $e^{16}$.

O estudo cefalométrico varia segundo a etnia. No nosso meio, devido à grande miscigenação racial, as falhas na interpretação podem ser mais contundentes.

O resultado de ambos os estudos, embora limitado pela condição de "paciente acordado", contribui para o aprimoramento diagnósticó ${ }^{4}$.

O sucesso do tratamento muitas vezes implica em alterações drásticas do modo de vida, e a adesão do paciente é difícil de ser conseguida nesses casos. A redução de peso corporal, a suspensão de medicamentos como os benzodiazepínicos, a abstenção de bebida alcoólica e a própria mudança no hábito de dormir em decúbito dorsal são exemplos ${ }^{12,14}$. Em relação à obesidade, questiona-se, até, se pode ser causa ou conseqüência dos distúrbios do sono ${ }^{17}$.

O uso de AIO pode ser incômodo por promover dores musculares ou na articulação têmporo-mandibular ${ }^{8}$.

O CPAP pode não ser usado adequadamente em decorrência de efeitos colaterais ${ }^{8,12}$.

Os resultados de procedimentos cirúrgicos são muitas vezes de valor duvidoso. Podem promover melhora inicial, com recidiva dos sintomas posteriormente ${ }^{18,19}$. Outros pacientes podem não apresentar qualquer melhora.

O procedimento cirúrgico mais realizado é a UPFP. Entretanto, nenhum método diagnóstico é capaz de predizer corretamente seu sucesso, sendo considerado efetivo somente em $50 \%$ dos $\operatorname{casos}^{18,20-22}$

Em nossa casuística, predominou a indicação de tratamento cirúrgico, na proporção de 2:1. Em realidade, a maioria dos pacientes da casuística tinha indicação de alguma modalidade cirúrgica.

A indicação de tratamento cirúrgico comparada à de tratamento não-cirúrgico se fez na mesma proporção nos casos de roncopatia. Nenhum dos 11 pacientes com roncopatia era obeso e o PAS estava diminuído em sua maioria (oito pacientes), ratificando o conceito de ser a faringe o principal sítio anatômico de obstrução. A desobstrução nesse local, contudo, não tem de ser necessariamente cirúrgica nas roncopatias.

$\mathrm{Na}$ SAHOS, a indicação de tratamento cirúrgico foi predominante em suas 3 modalidades (SAHOS leve $=2: 1$; SAHOS moderada $=3: 1$ e SAHOS grave $=3: 1$ ). Pinto $\&$ Colombini ${ }^{23}$ referem que pacientes portadores de SAHOS moderada e grave, em bom estado geral, devem preferencialmente ser submetidos a tratamento cirúrgico. Segundo Piccirillo et al. ${ }^{13}$, a cirurgia deveria ser o tratamento preferencial de roncadores e portadores de SAHOS leve. Nos casos de SAHOS moderada e grave, seria indicada somente em pacientes que recusam o CPAP. Vale sempre lembrar que IDR > 30 é considerado de mau prognóstico para a maioria das cirurgias ${ }^{24}$. O tratamento cirúrgico, contudo, não se constitui necessariamente no tratamento definitivo. Muitas vezes deverá ser complementado com outras intervenções clínicas, se não cirúrgicas mesmo.

O tratamento cirúrgico mais freqüentemente indicado foi UPFP, particularmente na SAHOS, independentemente do seu grau de severidade. Na literatura, encontramos referência de melhores resultados da UPFP em pacientes com SAHOS leve e moderado, embora a cirurgia, somente, possa não ser curativa ${ }^{25-27}$. O IDR $<30$ é um fator preditivo positivo de melhores resultados da UPFP ${ }^{28}$.

$\mathrm{Na}$ roncopatia, prevaleceram indicações cirúrgicas envolvendo a úvula e/ou palato mole adjacente ${ }^{4,26,29}$.

Houve predomínio de indicação cirúrgica que implica na atuação direta sobre tecidos moles redundantes (UPFP, LAUP, uvulectomia), em relação às atuações indiretas visando à abertura do espaço respiratório (traqueostomia, levantamento do hióide). Atualmente, a proposta de tratamento cirúrgico tende a ser a de procedimento complementar segundo a necessidade de cada caso ${ }^{30}$.

Fatores obstrutivos nasais não desempenharam papel importante na indicação cirúrgica - 3 septoplastias combinadas. Da mesma forma, a configuração esquelética não o fez - uma indicação de avançamento mandibular, uma de levantamento do hióide e uma de traqueostomia. Cirurgia nasal isolada é de utilidade limitada e pode ser realizada como complemento às faringoplastias ou para diminuir a pressão e melhorar a tolerância ao $\mathrm{CPAP}^{31-33}$. Cirurgia de avançamento mandibular é indicada em retrognatismo e o levantamento do hióide anterioriza a língua sem movimentação da mandỉbula ${ }^{34}$. Traqueostomia é indicada em SAHOS severa com comprometimento do estado geral e que não responde a outros procedimentos menos agressivos ${ }^{4,13,14,35,36}$.

Os tratamentos não-cirúrgicos mais indicados foram mudanças comportamentais e AIO.

Perda de peso é considerada de importância fundamental nos pacientes com IMC elevado, podendo reduzir ou, até mesmo, curar a SAHOS. Deve ser encorajada em todos os pacientes obesos ${ }^{12,14,29,37}$. Abstenção de álcool previne a exacerbação da $\mathrm{SAHOS}^{4}$ e o decúbito dorsal a agrava ${ }^{12}$. Alguns pacientes apresentam apnéia somente nesse decúbito ${ }^{38}$. 
Em pacientes com ronco primário e SAHOS leve que não respondem ou não têm indicação de mudanças comportamentais, indica-se o uso de $\mathrm{AIO}^{24}$. Este pode, ainda, ser indicado em SAHOS moderada e severa, nos pacientes que recusam tratamento com CPAP ou sem condições de tratamento cirúrgico ${ }^{39}$. Em dois pacientes, as condições clínicas impediam, transitória ou definitivamente, cirurgia sob anestesia geral. Indicou-se o CPAP. O uso de CPAP é um tratamento eficaz, mas deve-se ter em mente que ele, ao não curar o distúrbio ventilatório, deve ser usado durante toda a vida ${ }^{13,14,18,40}$.

Em 2 deles encontramos alterações neurológicas subclínicas, expressando-se por apnéia mista, optando-se pelo tratamento medicamentoso.

O IMC não teve influência na indicação de tratamento não-cirúrgico, quer quanto à sua modalidade, quer na relação desta com o distúrbio ventilatório apresentado pelo paciente.

Encontramos maior proporção de obesos entre os pacientes com SAHOS grave, quando comparados aos não obesos (2,5:1). Mesmo entre estes, predominou a indicação de UPFP. Em geral, UPFP é o procedimento cirúrgico mais indicado. Entretanto, o ganho de peso no pós-operatório pode prejudicar o resultado inicial ${ }^{26}$.

Embora estudo de Doghramji et al ${ }^{22}$ indique que a radiografia cefalométrica e a manobra de Müller não possam ser usadas confiavelmente para predizer o sucesso cirúrgico da UPFP, até o presente momento estes dois métodos parecem ser os mais úteis ${ }^{27}$. Muitos autores consideram que na SAHOS há comprometimento difuso da via aérea, mais do que um processo localizado. Nenhum método seria capaz de predizer corretamente o sucesso na sua correção cirúrgi$\mathrm{ca}^{18,41,42}$. Segundo Metes et $\mathrm{al}^{42}$, mesmo tendo sido identificada a região retropalatal como o local da obstrução, esta localização não prediz o sucesso da UPFP.

Millman et al. ${ }^{28}$ realizaram estudo para determinar se polissonografia, cefalometria e dados antropométricos poderiam predizer o sucesso ou o insucesso da UPFP. Fizeram estudo retrospectivo de 46 pacientes submetidos a esta cirurgia. Concluíram que IDR < 38 e $\mathrm{Mp}-\mathrm{H} \leq 20 \mathrm{~mm}$, além de ausência de retrognatia, foram preditivos de sucesso cirúrgico.

As medidas PNS-P e MP-H encontradas estão predominantemente acima da média citada na literatura $(93,3 \%$ e $96,7 \%$ dos casos respectivamente). Embora a comparação se faça com dados não próprios da nossa raça, são fortemente sugestivos de indicadores de diminuição da área residual orofaríngea e conseqüente redução da permeabilidade da coluna aérea, fortalecendo a idéia de indicação de tratamento cirúrgico predominantemente. São achados comparáveis aos descritos por FARIA ${ }^{43}$, em sua dissertação de tese de mestrado.

Da mesma forma, houve predomínio na indicação de tratamento cirúrgico independentemente dos valores PAS <
11 ou PAS $\geq 11$. Em relação a PAS $<11$, encontramos indicação de tratamento cirúrgico comparada à de não-cirúrgico na proporção $1,6: 1$. Nos casos de PAS $\geq 11$, a mesma proporção foi de 5:1.

Quando comparamos tipo de distúrbio ventilatório com PAS, observamos maior ocorrência de SAHOS leve e SAHOS grave nos pacientes com PAS $<11$ (13 e 14 pacientes, respectivamente, no total de 42). Os demais se distribuem em oito pacientes com roncopatia e sete com SAHOS moderada. Quando ambas essas variáveis (distúrbio ventilatório e PAS < 11) são comparadas com IMC, encontramos 20 pacientes não-obesos e 22 obesos. O IMC não teve praticamente importância tanto no tipo de distúrbio ventilatório como na indicação de tratamento cirúrgico. Considerando-se que esses pacientes tiveram indicação de tratamento cirúrgico versus tratamento não-cirúrgico na proporção de 1,6:1, fica-nos a impressão de que nenhuma das variáveis estudadas teve papel preponderante na modalidade de tratamento sugerida.

Os pacientes com PAS $\geq 11$ distribuem-se em: $3 \mathrm{com}$ roncopatia, 7 com SAHOS leve, 1 com SAHOS moderada e 7 com SAHOS grave. Quando comparados com o IMC, encontramos 11 não-obesos e 7 obesos. Como nesses casos predominou a indicação de tratamento cirúrgico, cuja modalidade mais freqüente foi a UPFP, parece-nos que o volume da tonsila palatina seja um aspecto importante na decisão por essa modalidade terapêutica. O efeito de massa, resultante desse órgão hipertrofiado, seria o responsável pelo PAS paradoxalmente aumentado, tendo em vista a ocorrência de fenômeno obstrutivo importante.

Encontramos predominância da manobra de Müller positiva nos 2 níveis (23 pacientes), em relação à negativa em ambos ( 7 pacientes), estabelecendo-se a proporção de $3,3: 1$.

Entre os pacientes com manobra de Müller positiva nos 2 níveis, encontramos 4 com roncopatia, 8 com SAHOS leve, 2 com SAHOS moderada e nove com SAHOS grave. Em relação ao IMC, 12 eram obesos e 11 não-obesos. Encontramos 15 pacientes com PAS $<11$ e 8 com PAS $\geq 11$, na proporção de 1,4:1. Nesses pacientes, indicou-se cirurgia em 16, sendo a UPFP indicada em 9 deles. Sete pacientes tiveram indicação de tratamento não-cirúrgico, em que predominou a indicação de AIO (4 pacientes). Dois tiveram indicação de CPAP.

A positividade da manobra de Müller em 2 sítios anatômicos não parece ter relação com a natureza do processo obstrutivo nem com o IMC. Por outro lado, parece reforçar a necessidade de expansão das vias aéreas na rinofaringe e orofaringe/hipofaringe, quer cirurgicamente, quer sob a forma de AIO como primeira medida a tomar-se.

Entre os negativos nos 2 níveis, encontramos $1 \mathrm{com}$ roncopatia, 3 com SAHOS leve, 1 com SAHOS moderada e 2 com SAHOS grave. Em relação ao IMC, 3 eram obesos e 4 não-obesos. Encontramos 5 pacientes com PAS $<11$ e 2 com 
PAS $\geq 11$. Nesses pacientes, indicou-se cirurgia em 6 , sendo 3 UPFP e 3 uvulectomia.

A despeito da negatividade da manobra de Müller em 2 sítios, deparamos com indicações que visam expandir o espaço disponível na rinofaringe e orofaringe/hipofaringe através dos 2 tipos de cirurgias mais indicadas.

Entre os 23 pacientes com Müller positivo só na rinofaringe, encontramos 4 com roncopatia, 7 com SAHOS leve, 4 com SAHOS moderada e 8 com SAHOS. Em relação ao IMC, 11 eram obesos e 12 não-obesos. Encontramos 18 pacientes com PAS $<11$ e 5 com PAS $\geq 11$. Nesses pacientes, indicou-se cirurgia em 13, sendo 10 UPFP. Nos 10 pacientes com indicação de tratamento não-cirúrgico, predominou a indicação de mudanças comportamentais (5 pacientes). Dois tiveram indicação de tratamento medicamentoso e 3 tiveram indicação de AIO.

Entre os 7 pacientes com Müller positivo só na orofaringe/hipofaringe, encontramos 2 com roncopatia, 2 com SAHOS leve, 1 com SAHOS moderada e 2 com SAHOS grave. Em relação ao IMC, 3 eram obesos e 4 não-obesos. Encontramos 4 pacientes com PAS $<11$ e 3 com PAS $\geq 11$. Nesses pacientes, indicou-se cirurgia em 6 , sendo 5 UPFP.

\section{CONCLUSÕES}

1. A modalidade de tratamento inicial mais indicada foi a cirúrgica.

2. Nas roncopatias, a indicação de tratamento cirúrgico e não-cirúrgico se fez na mesma proporção.

3. A indicação de tratamento cirúrgico prevaleceu na SAHOS, independentemente de sua modalidade.

4. O tratamento cirúrgico mais freqüentemente indicado foi UPFP.

5. Predominou a indicação de cirurgias que intervêm diretamente sobre tecidos moles da faringe (UPFP, LAUP, uvulectomia, amigdalectomia).

6. Os tratamentos não-cirúrgicos mais freqüentemente indicados foram alterações comportamentais e AIO.

7. A indicação de CPAP restringiu-se a pacientes sem condições de submeter-se a tratamento cirúrgico, temporária ou definitivamente.

8. O IMC não teve influência na indicação de qualquer modalidade de tratamento.

9. O estudo cefalométrico não foi importante na opção por tratamento cirúrgico ou não-cirúrgico.

10. Na comparação entre modalidade de distúrbio ventilatório, PAS < 11 e IMC, nenhuma das variáveis teve papel preponderante na opção por tratamento cirúrgico ou não-cirúrgico.

11. A opção preponderante por tratamento cirúrgico, quando da comparação entre modalidade de distúrbio ventilatório, PAS $\geq 11$ e IMC, mostra a importância do volume amigdaliano na gênese do fenômeno obstrutivo e seu papel no "aumento paradoxal" do espaço posterior no estudo cefalométrico.

12. A manobra de Müller não interferiu diretamente na opção por tratamento cirúrgico, mesmo quando negativa em ambos os sítios anatômicos estudados.

13. A decisão terapêutica, sem dúvida, deve decorrer de estudo propedêutico sistematizado e de atuação multidisciplinar, havendo a necessidade de que cada caso seja individualmente discutido.

\section{AGRADECIMENTOS}

Agradeço aos Profs. Drs. Manoel Baldoíno Filho e Benedito Borges da Silva pela orientação na síntese deste artigo.

\section{REFERÊNCIAS BIBLIOGRÁFICAS}

1. Redline S, Tishler PV. The genetics of sleep apnea. Sleep Med Rev 2000; 4 (6):583-602.

2. Woodson BT, Ledereich PS, Strollo P. Obstructive sleep apnea syndrome: diagnosis and treatment. In: American Academy of Otolaryngology - Head and Neck Surgery Foundation, Inc. 1996; 1. Ed. Rochester, Minnesota, Custom Printing, Inc., p.13.

3. Casero MAR, Rio FG, Garcia JMP, Sánchez CP, Lobato SD, Leon JV. El síndrome de apneas durante el sueño como problema sanitario. Valoración de su prevalencia y morbimortalidad. An. Med. Intern (Madrid) 1999; 16 (2):97-102.

4. Barthel SW, Strome M. Snoring, obstructive sleep apnea, and surgery. Med Clin North Am 1999; 83 (1):85-96.

5. Pieters T, Rodenstein DO. Therapeutic options in obstructive sleep apnoea: have we made enough progress? Sleep Med Ver 2001; 5 (1):3-6. [Editorial]

6. Kapur V, Blough DK, Sandblom RE, Hert R, Maine JB, Sullivan SD, Psaty BM. The medical cost of undiagnosed sleep apnea. Sleep 1999; 22(6):749-55.

7. Rosen CL. Obstructive sleep apnea syndrome (OSAS) in children: diagnostic challenges. Sleep 1996; 19(suppl. 10):S274-S277.

8. Sériès F. Evaluation of treatment efficacy in sleep apnea hypopnea syndrome. Sleep, 19 1996; (suppl. 9):S71-S76.

9. Riley R, Guilleminault C, Herran J, Powell N. Cephalometric analyses and flow-volume loops in obstructive sleep apnea patients. Sleep 1983; 6(4):303-11.

10. Alvarez CM, Lessin ME, Gross PD. Mandibular advancement combined with horizontal advancement genioplasty for the treatment of obstructive sleep apnea in an edentulous patient. Oral Surg Oral Med Oral Pathol 1987; 64:402-6.

11. American Academy Of Sleep Medicine Task Force. Sleep-related breathing disorders in adults: recommendations for syndrome definition and measurement techniques in clinical. Sleep 1999; 22(5):667-8

12. Lévy P, Pétin JL, Mayer P, Wuyam B, Veale D. Management of simples snoring, upper airway resistance syndrome and moderate sleep apnea syndrome. Sleep 1996; 19 (suppl. 9): S101-S110.

13. Piccirillo JF, Duntley S, Schotland H. Apnéia Obstrutiva do Sono. JAMA Brasil 2001; 5(2):134-7.

14. Man GCW. Obstructive sleep apnea - diagnosis and treatment. Medical Clinics of North America 1996; 80(4):803-21.

15. Skomro RP, Kryger MH. Clinical presentation of obstructive sleep apnea syndrome. Progress in Cardiovascular Diseases 1999; 41(5):331-40.

16. Quinn SJ, Daly N, Ellis PDM. Observation of the mechanism of snoring using sleep nasendoscopy. Clin. Otolaryngol 1995; 20: $360-4$. 
17. Simmons FB, Guilleminaut C, Dement WC, Tilkian AG, Hill M Surgical management of airway obstructions during sleep. Laryngoscope 1976; 87:326-38.

18. Pépin JL, Veale D, Mayer P, Bettega G, Wuyam B, Lévy P. Critical analysis of the results in the treatment of snoring, upper airway resistance syndrome and obstructive sleep apnea. Sleep 1996; 19 (suppl. 9):S90-S100.

19. Boot H, Wegen RV, Poublon RML, Bogaard JM, Schmitz PIM, Meché FGAVD. Long-term results of uvulopalatopharyngoplasty for obstructive sleep apnea syndrome. Laryngoscope 2000; 110: 469-75.

20. Fugita S. Surgical correction of anatomic abnormalities in obstructive sleep apnea syndrome: uvulopalatopharyngoplasty. Otolaryngol Head Neck Surg 1981; 89:923-34.

21. Fugita S. UPPP for sleep apnea and snoring. Ear, Nose and Throat Journal 1984; 63:73-86.

22. Doghramji K, Jabourian ZH, Pilla M, Farole A, Lindholm RN. Predictors of outcome for uvulopalatopharyngoplasty. Laryngoscope 1995; 105:311-4.

23. Pinto JÁ, Colombini NEP. Projeto de um protocolo para tratamento dos distúrbios respiratórios dependentes. In: Pinto JA. Ronco e apnéia do sono 2000; 1. Ed. Rio de Janeiro: Livraria e Editora Revinter. p. 227-65.

24. Sociedade Brasileira de Sono. Consenso Brasileiro em Ronco e Apnéia do Sono. Hypnos 2001; (suppl. 1):21-2.

25. Katsantonis GP, Friedman WH, Rosenblum BN, Walsh JK. The surgical treatment of snoring: a patient's perspective. Laryngoscope $1990 ; 100: 138-40$.

26. Friberg D, Norlander BC, Larsson H, Svanborg E. UPPP for habitual snoring: 5-year follow-up with respiratory sleep recording. Laryngoscope 1995; 105(5):519-22.

27. Sher AE, Schechtman KB, Piccirillo JF. An American Sleep Disorders Association Review: the efficacy of surgical modifications of the upper airway in adults with obstructive apnea syndrome. Sleep, 19 1996; (2):156-77.

28. Millman RP, Carlisle CC, Rosenberg C, Kahn D, McRae R, Kramer NR. Simple predictors of uvulopalatopharyngoplasty outcome in the treatment of obstructive sleep apnea. Chest 2000; 118(4): 1025-30.

29. Lindblom SS. Adults who snore: social nuisance or at risk for medical problems? Postgraduate Medicine 1997; 101(6):171-91.

30. Riley RW, Powell NB, Guilleminault C. Obstructive sleep apnea syndrome: a review of 306 consecutively treated surgical patients Otolaryngology- Head and Neck Surgery 1993; 108:117-25.
31. Utley DS, Shin EJ, Clerk AA, Terris DJ. A cost-effective and rational surgical approach to patients with snoring, upper airway resistance syndrome, or obstructive sleep apnea syndrome. Laryngoscope 1997; 107:726-34

32. Powell NB, Riley RW, Robinson A. Surgical management of obstructive sleep apnea syndrome. Clinics in Chest Medicine 1998; 19(1):77-86.

33. Friedman M, Tanyeri H, Lim JW, Landsberg R, Vaidyanathan $\mathrm{K}$, Caldarelli D. Effect of improved nasal breathing on obstructive sleep apnea. Otolaryngol Head Neck Surg. 2000, 122(1):71-4.

34. Sher AE. The role of maxillomandibular surgery for treating obstructive sleep apnea. Sleep, 19 1996; (suppl. 9):S88-S89.

35. Woodson BT, Fugita S. Clinical experience with lingualplasty as part of the treatment of severe obstructive sleep apnea. Otolaryngology-Head and Neck Surgery 1992; 107(1):40-8.

36. Carney AS, Robinson PJ. Assessment and management of snoring: a surgical perspective. Br J Hosp Med 1995; 53(10):515-21.

37. Suratt PM, McTier RF, Findley LJ, Pohl SL, Wilhoit SC. Changes in breathing and the pharynx after weight loss in obstructive sleep apnea. Chest 1987; 92(4):631-7.

38. Cartwright R, Ristanovic R, Diaz F, Caldarelli D, Alder G. A comparative study of treatments for positional sleep apnea. Sleep $1991 ; 14(6): 546-52$.

39. American Sleep Disorders Association Report. Practice parameters for the treatment of snoring and obstructive sleep apnea with oral appliances. Sleep 1995; 18(6):511-3.

40. Kribbs NB, Pack AI, Kline LR, Getsy JE, Schuett J.S, Henry JN, Maislin G, Dinges DF. Effects of one night without nasal CPAP treatment on sleep and sleepiness in patients with obstructive sleep apnea. Am. Rev. Respir. Dis 1993; 147:1162-8.

41. Katsantonis GP, Maas C.S, Walsh JK. The predictive efficacy of the Muller Maneuver in uvulopalatopharyngoplasty. Laryngoscope 1989; 99:677-80.

42. Metes A, Hoffstein V, Mateika S, Cole P, Haight JSJ. Site of airway obstruction in patients with obstructive sleep apnea before and after uvulopalatopharyngoplasty. Laryngoscope 1991; 101:11028.

43. Faria AC. Estudo cefalométrico em pacientes com distúrbios ventilatórios obstrutivos do sono. São Paulo, 2002. [Tese - Mestrado - Faculdade de Ciências Médicas da Universidade Estadual de Campinas]. 